\title{
Advances in ultrasound diagnosis in chronic liver diseases
}

\author{
Hitoshi Maruyama and Naoya Kato \\ Department of Gastroenterology, Chiba University Graduate School of Medicine, Chiba, Japan
}

Chronic liver disease is a major disorder worldwide. A better understanding of anatomy, blood flow, and pathophysiology may be a key issue for their proper management. Ultrasound (US) is a simple and non-invasive diagnostic tool in the abdominal field. Doppler mode offers real-time hemodynamic evaluation, and the contrast-enhanced US is one of the most frequently used modalities for the detailed assessment. Further development in digital technology enables threedimensional (3D) visualization of target images with high resolution. This article reviews the wide ranges of application in the abdominal US and describes the recent progress in the diagnosis of chronic liver diseases. (Clin Mol Hepatol 2019;25:160-167)

Keywords: Portal hypertension; Fibrosis; Cirrhosis; Contrast agent

\section{INTRODUCTION}

With the various spectrums of the etiologies, chronic liver disease is increasing in prevalence worldwide.' Cirrhosis is the most advanced stage of chronic liver disease. It is associated with possible adverse events, such as gastroesophageal varices, ascites, hepatic encephalopathy, and the development of hepatocellular carcinoma ( $\mathrm{HCC}$ ), which require careful medical care. ${ }^{2-4}$

Because of the advantages of simple and less-invasive evaluations, ultrasound (US) may be the most frequently used imaging tool in the practical management of patients with chronic liver disease. In addition to the B-mode imaging, recent development in digital technology has introduced various modes, color/power Doppler mode, harmonic imaging as a microbubble-based contrast mode, and three-dimensional (3D) mode. ${ }^{5-7}$ With these back- grounds, this review article describes the recent advances of US in the diagnosis of chronic liver diseases.

\section{B-MODE}

Recent digital development has shown the improvement of spatial resolution and signal-to-noise ratio of B-mode which is the only imaging to demonstrate fundamental tissue images. The role of simple technique for chronic liver disease is identification of cirrhosis, diameter measurement (i.e., liver, spleen, and vessels), and detection of the ascites, collateral vessels, and focal hepatic lesions. $^{8-10}$

Muscle is another target of B-mode US. The quantification of muscle mass is an important issue of patient care because it is

\section{Abbreviations: \\ APRI, aspartate aminotransferase to platelet ratio index; AUROC, area under the receiver operating characteristic; $C T$, computed tomography; ELF, enhanced liver fibrosis; EV, esophageal varices; FIB-4, fibrosis index based on 4 factors; GV, gastric varices; HCC, hepatocellular carcinoma; HVAT, transit time from venous access to the hepatic vein; HVPG, hepatic venous pressure gradient; IP, iliopsoas muscle; IPH, idiopathic portal hypertension; LGV, left gastric vein; MRI, magnetic resonance imaging; NFPF, non-forward portal flow; NPV, negative predictive value; $\mathrm{SPH}$, severe portal hypertension; TE, transient elastography; US, ultrasound; 3D, three-dimensional}

\section{Corresponding author: Hitoshi Maruyama}

Department of Gastroenterology, Chiba University Graduate School of Medicine, 1-8-1 Inohana, Chuou-ku, Chiba 260-8670, Japan

Tel: +81-43-2262083, Fax: +81-43-2262088

E-mail: maru-cib@umin.ac.jp

https://orcid.org/0000-0003-3371-3157 
closely associated with malnutrition, leading to the reduction of health-related quality of life. ${ }^{11,12}$ Sarcopenia is defined by significant reduction of muscle mass and/or power and is widely accepted as a common impairment in cirrhosis. ${ }^{13,14}$ It is a significant factor for poor prognosis, for prediction of outcome in patients with HCC, and for the development of complications after hepatic surgery or liver transplantation. ${ }^{15-17}$ A study based on the B-mode US reported that the detectability of the iliopsoas muscle (IP) was $100 \%$ both in the control group (depth 31.7-61.2 mm; mean \pm standard deviation, 45.8 \pm 7.7$)$ and in the cirrhosis group (20.6$75.4 \mathrm{~mm} ; 43.7 \pm 9.2$ ) (Fig. 1). Sensitivity, specificity, and the area under the receiver operating characteristic (AUROC) by IP index (IP area/height ${ }^{2}, \mathrm{~mm}^{2} / \mathrm{m}^{2}$ ) calculated by US to detect muscle mass loss diagnosed by computed tomography (CT) using skeletal muscle index at L3 level were $79.5 \%, 73.1 \%$, and 0.835 , respectively, with the best cut-off value of 189.2 for males, and $84.6 \%$, $78.8 \%$, and 0.874 , respectively, with the best cut-off value of 180.6 for females. ${ }^{18}$ Thus, the IP index obtained from the transcutaneous US may be an alternative to $\mathrm{CT}$ the muscle mass quantification in cirrhosis, without requiring radiation exposure.

Recent development of digital technology has resulted in the demonstration of 3D hepatic surface sonogram with high-qualified images and real-time manner; $74 \%$ (23/31) success rates of visualization showing characteristic irregularity of the hepatic surface in all cirrhotic patients and the intra-/inter-operator and interreviewer agreement were excellent $(\mathrm{\kappa}=1.0)^{19}{ }^{19}$ The ability to distinguish cirrhotic liver from non-cirrhotic liver improved with the use of combination of 2D-and $3 \mathrm{D}$-imaging versus $2 \mathrm{D}$-imaging alone (sensitivity, $P=0.02$; accuracy, $P=0.02$ ) or 3D-imaging alone (sensitivity, $P=0.03$ ). Therefore, 3D-based sonography may act as a virtual laparoscopy method with the potential to improve the diagnosis of cirrhosis.

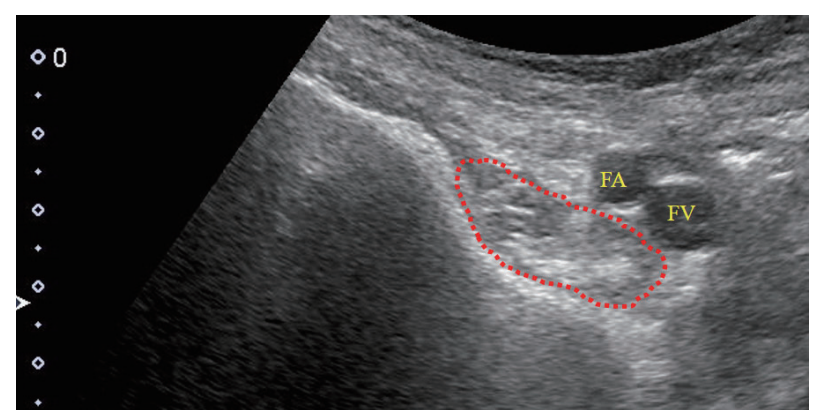

Figure 1. An 84-year old female, cirrhosis (primary biliary cholangitis). Bmode sonogram (transverse scan) at right groin area showed iliopsoas muscle with clear delineation (red dotted line). FA, femoral artery; FV, femoral vein.

\section{DOPPLER US}

Doppler US is the first-line modality for the diagnosis of vascular-related liver diseases, ${ }^{20}$ with possible evaluation of waveform, flow direction, a velocity and flow volume. A damping index is a parameter using the hepatic vein waveform calculated by the following formula: minimum velocity/maximum velocity of the hepatic vein waveform. If the value was higher than 0.6 , the patient is significantly more likely to have severe portal hypertension $(\mathrm{SPH}$; hepatic venous pressure gradient [HVPG] $>12 \mathrm{mmHg}$ ), with $76 \%$ sensitivity and $82 \%$ specificity, suggesting it to be an effective parameter to predict the grade of portal hypertension. ${ }^{21}$

Flow direction is also the target of Doppler US observation. The study in 222 cirrhosis patients reported that the cumulative incidences of non-forward portal flow (NFPF) were $6.5 \%$ at 1 year, $14.5 \%$ at 3 years, and $23.1 \%$ at 5 years. The cumulative survival rate was significantly lower in patients with NFPF $(72.2 \%$ at 1 year, $38.5 \%$ at 3 years, and $38.5 \%$ at 5 years) than in those with forward portal flow $(84.0 \%$ at 1 year, $67.8 \%$ at 3 years, and $54.3 \%$ at 5 years; $P=0.0123$ ) on using Child-Pugh $B$ and $C$ classification, suggesting the need for careful management. ${ }^{22}$

It is also reported that portal hemodynamics are predictive markers of outcomes in cirrhosis; lower velocity $(<12.8 \mathrm{~cm} / \mathrm{s})$ in the portal trunk in compensated cirrhosis for decompensation, and reverse portal flow in decompensated cirrhosis for poor prognosis. $^{23}$

\section{ESOPHAGEAL VARICES (EV)}

Based on the anatomical and hemodynamic features, left gastric vein (LGV) is the key vascular route to the EV (Fig. 2). A recent study reported that the sonographic detectability of the LGV was higher in patients with EV $(129 / 154,83.8 \%)$ than in those without $(35 / 75,46.7 \% ; P<0.0001)$, and was higher in those with large $\operatorname{EV}(30 / 30,100 \%)$ than in those without $(134 / 199,67.3 \%$; $P=0.0002) .{ }^{24}$ The positive detection of the LGV showed $100 \%$ sensitivity and negative predictive value (NPV) to identify large EV in the whole cohort and the compensated group. The best cutoff value in the LGV diameter was $5.35 \mathrm{~mm}$ to identify large $E V$, showing 0.753 AUROC with $90 \%$ sensitivity and $96.5 \%$ NPV. It may enhance the practical use of US-based LGV assessment as a noninvasive test to identify large EV with high sensitivity and NPV in cirrhotic patients at a lower cost. 


\section{GASTRIC VARICES (GV)}

GV are one of the serious complications in cirrhosis. Although the frequency is relatively lower than that of $\mathrm{EV}$, bleeding from GV may result in much severer consequence. ${ }^{25}$ Needless to say, presence and degree of GV need to be properly recognized for their clinical management. The 3D color Doppler may be effective for the quantitative evaluation of the degree of GV; the sensitivity and specificity of the 3D-US technique to detect GV were $88.6 \%$ $(62 / 70)$ and $100 \%$ (37/37), respectively. The best cutoff value of variceal volume to detect medium/large-grade GV was $2.0 \mathrm{~mL}$, with $83.3 \%$ sensitivity and $95.8 \%$ specificity. Seventy-nine percent (55/70) of patients showed partial resemblance or better between the 3D images and the endoscopic findings with the good inter-reviewer agreement. These data suggest the improvement of objectivity in the assessment of GV by using 3D-US, which is expected to be an alternative to endoscopy. ${ }^{26,27}$

With regard to the hemodynamic aspect, Doppler US is effective to evaluate the inflow and outflow route of GV for the assessment of bleeding risk (Fig. 3). ${ }^{28,29}$ Particularly, demonstration of the gastrorenal shunt may be useful to decide the therapeutic strategy because it is a key pathway to perform balloon-occluded retrograde transvenous obliteration. Hemodynamic evaluation by Doppler US also offers the prediction of long-term prognosis after treatment of GV; large flow volume in the collaterals and reverse flow direction of splenic vein suggest the post-treatment poor prognosis. ${ }^{30,31}$
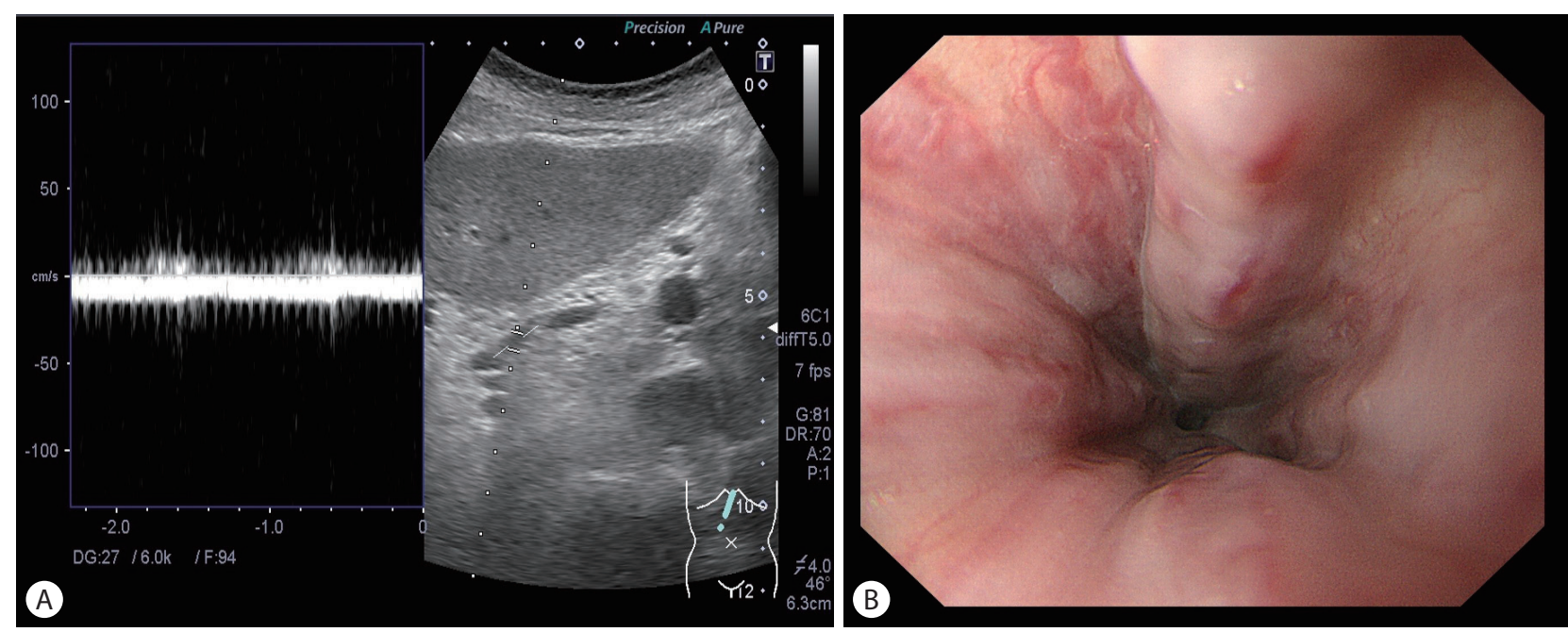

Figure 2. A 46-year old female, alcoholic cirrhosis. (A) Pulsed Doppler image. Left gastric vein showed hepatofugal flow direction. (B) Endoscopic finding. The patient had medium-grade esophageal varices with a red sign.
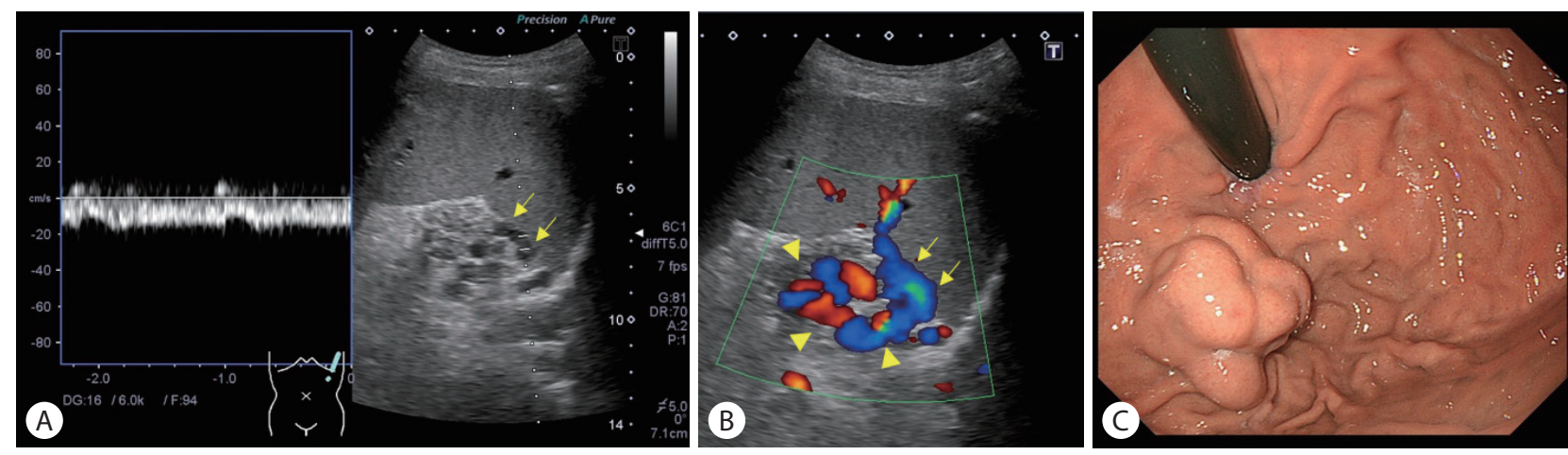

Figure 3. A 25-year old male, non B non C cirrhosis. (A) Pulsed Doppler image. Short gastric vein (arrows) had a continuous wave with hepatofugal flow direction. (B) Color Doppler image. The stomach was demonstrated through the spleen (arrows, short gastric vein; arrowheads, gastric varices). (C) Endoscopic finding. The patient had medium-grade gastric fundal varices. 


\section{CONTRAST-ENHANCED US}

\section{Hepatic fibrosis}

\section{Transit time}

The transit time is defined by the interval from vessel to vessel and represents the parameter for evaluating dynamic microbubbles (Table 1). ${ }^{32,33}$

A prospective multicenter study reported the effect of the transit time from the portal vein to the hepatic vein using SonoVue: $78.6 \%$ specificity, $79 \%$ sensitivity, $78.3 \%$ positive predictive value, $83.3 \%$ NPV, $78.8 \%$ accuracy, and 0.847 AUROC at a cutoff of 13 seconds for the diagnosis of severe fibrosis (F3 and F4) with various etiologies. ${ }^{34}$ A more recent study with the same parameter using SonoVue in chronic hepatitis B virus infection has shown AUROCs of 0.955 for $\geq S 2$ (Scheuer scoring system) and 0.946 for $\geq S 4$, with better diagnostic ability than Fibrolndex. ${ }^{35}$ However, Cobbold et al. reported poor diagnostic ability of transit time (hepatic artery to hepatic vein) in patients with chronic hepatitis $\mathrm{C}$ virus infection using SonoVue: AUROCs of 0.83 with aspartate aminotransferase to platelet ratio index (APRI), 0.82 with enhanced liver fibrosis (ELF)/transient elastography (TE), 0.71 with hepatic transit time for moderate to severe fibrosis (stage $>2$ ), 0.91 with ELF, 0.9 with TE, 0.86 with APRI, and 0.83 with hepatic transit

Table 1. Contrast-enhanced parameters for hepatic fibrosis

\begin{tabular}{|c|c|c|c|c|c|c|}
\hline Contrast agent & $N$ & Parameter (cut-off value) & Grade of fibrosis & $\mathrm{Se} / \mathrm{Sp} / \mathrm{PPV} / \mathrm{NPV} / \mathrm{Ac}$ & $A z$ & Study \\
\hline SonoVue & 99 & PV to HV (13 s) & Severe fibrosis (F3-4) & 79/79/73/83/79 & 0.847 & Ref. 34 \\
\hline \multirow[t]{2}{*}{ SonoVue } & 134 & $\begin{array}{l}\text { PV to HV } \\
(6 s) \\
(4.12 s)\end{array}$ & $\begin{array}{l}\geq \text { S2 (Scheuer scoring) } \\
\quad \text { Cirrhosis }\end{array}$ & $\begin{array}{l}83 / 97 /-/-193 \\
87 / 89 /-/-/ 88\end{array}$ & $\begin{array}{l}0.955 \\
0.946\end{array}$ & Ref. 35 \\
\hline & & $\begin{array}{l}\text { HA to HV } \\
(9.63 \mathrm{~s}) \\
(8.13 \mathrm{~s})\end{array}$ & $\begin{array}{l}\geq \text { S2 (Scheuer scoring) } \\
\quad \text { Cirrhosis }\end{array}$ & $\begin{array}{l}75 / 95 /-/-/ 89 \\
60 / 84 /-/-/ 72\end{array}$ & $\begin{array}{l}0.891 \\
0.785\end{array}$ & \\
\hline SonoVue & 67 & $\begin{array}{l}\text { HA to HV } \\
(10.25 \mathrm{~s}) \\
(8.0 \mathrm{~s})\end{array}$ & $\begin{array}{c}\text { Moderate to severe } \\
\text { (>2, Ishak fibrosis stage) } \\
\text { Cirrhosis }\end{array}$ & $\begin{array}{l}\text { 53/73/62/66/- } \\
71 / 91 / 67 / 92 /-\end{array}$ & $\begin{array}{l}0.71 \\
0.83\end{array}$ & Ref. 36 \\
\hline \multirow[t]{2}{*}{ SonoVue } & 75 & $\begin{array}{l}\text { HV arrival time } \\
(25.25 \mathrm{~s}) \\
(23.75 \mathrm{~s})\end{array}$ & $\begin{array}{c}\text { Moderate to severe } \\
\text { (Ishak stages 3-6) } \\
\text { Cirrhosis (Ishak stages 5-6) }\end{array}$ & $\begin{array}{l}80 / 57 / 68 / 71 /- \\
82 / 40 / 29 / 88 /-\end{array}$ & $\begin{array}{l}0.72 \\
0.71\end{array}$ & Ref. 33 \\
\hline & & $\begin{array}{l}\text { HA to HV } \\
(10.25 \mathrm{~s}) \\
(7.25 \mathrm{~s})\end{array}$ & $\begin{array}{c}\text { Moderate to severe } \\
\text { (Ishak stages 3-6) } \\
\text { Cirrhosis (Ishak stages 5-6) }\end{array}$ & $\begin{array}{l}80 / 60 / 70 / 72 /- \\
82 / 61 / 39 / 92 /-\end{array}$ & $\begin{array}{l}0.76 \\
0.78\end{array}$ & \\
\hline SonoVue & 59 & $\begin{array}{l}\text { Peak signal intensity }{ }^{*} \\
(16 \mathrm{~s})\end{array}$ & Severe fibrosis & 83/77/-/-/- & 0.88 & Ref. 39 \\
\hline Definity & 43 & $\begin{array}{l}\text { Venous access to HV } \\
\text { (24 s) }\end{array}$ & $\begin{array}{c}\text { Severe fibrosis } \\
\text { (F3.5-4.0, fibrosis score) }\end{array}$ & 57/43/17/83/- & 0.56 & Ref. 37 \\
\hline Sonazoid & 117 & $\begin{array}{l}\text { Peak intensity/time }{ }^{\dagger} \\
(6.5 \mathrm{~s}) \\
(8.0 \mathrm{~s}) \\
(9.5 \mathrm{~s})\end{array}$ & $\begin{array}{l}F 2 \\
\text { F3 } \\
\text { F4 }\end{array}$ & $\begin{array}{l}84 / 88 / 84 / 88 / 87 \\
83 / 93 / 89 / 90 / 89 \\
95 / 92 / 77 / 98 / 93\end{array}$ & $\begin{array}{l}0.94 \\
0.96 \\
0.98\end{array}$ & Ref. 40 \\
\hline Sonazoid & 203 & $\begin{array}{l}\text { Parenchymal intensity }{ }^{\ddagger} \\
(-) \\
(-) \\
(-)\end{array}$ & $\begin{array}{l}\text { F2 } \\
\text { F3 } \\
\text { F4 }\end{array}$ & $\begin{array}{l}88 / 72 / 78 / 84 / 81 \\
85 / 91 / 85 / 91 / 89 \\
97 / 90 / 70 / 99 / 91\end{array}$ & $\begin{array}{l}0.88 \\
0.95 \\
0.97\end{array}$ & Ref. 42 \\
\hline
\end{tabular}

Se, sensitivity; Sp, specificity; PPV, positive predictive value; NPV, negative predictive value; Ac, accuracy; Az, area under the receiver operating characteristic curve; PV, portal vein; HV, hepatic vein; Ref., reference; HA, hepatic artery.

"Peak signal intensity $(\mathrm{dB})$ means the difference in the peak intensity between in the portal vein and in the liver parenchyma.

${ }^{\dagger}$ Peak intensity/time means the time to the maximum intensity ratio between the right portal vein and liver parenchyma from the onset of contrast enhancement in the portal vein.

${ }^{\ddagger}$ Parenchymal Intensity means signal intensity from the accumulated microbubbles at the late phase ( $15 \mathrm{~m}$ after the agent injection). 
time for cirrhosis. ${ }^{36}$ The transit time from venous access to the hepatic vein (HVAT) using Definity showed an AUROC of only 0.56 in the differentiation of $\geq \mathrm{F} 3$. $^{37}$ The other study suggested no significant effect of HVAT on METAVIR scores of fibrosis and necroinflammatory changes in patients with chronic hepatitis $C^{38}$ Taken together, the efficacy of transit time to estimate the degree of hepatic fibrosis is controversial. An optimal time point with proper kind of contrast agents needs to be determined by further studies.

\section{Intensity-based parameter}

The signal intensity is also a popular parameter in the assessment of contrast sonography. The difference in the peak signal intensity between the portal vein and liver parenchyma showed an AUROC of 0.87 for $>F 3$ in 49 patients with hepatitis C virus infection using SonoVue. ${ }^{39}$ The interval between the portal onset and the time of maximum intensity ratio between the right portal vein and parenchyma showed a close relationship with the degree of hepatic fibrosis using Sonazoid in 117 chronic liver disease patients with AUROCs of 0.94 for $\geq F 2,0.96$ for $\geq F 3$, and 0.98 for cirrhosis. ${ }^{40}$ The diagnostic ability was superior to those of APRI and fibrosis index based on 4 factors (FIB-4). A subsequent study (in 74 patients with chronic liver disease) compared the diagnostic performance using the following 4 markers: contrast parameter (Sonazoid, the same contrast parameter used $\mathrm{in}^{40}$ ), TE, type IV collagen $7 \mathrm{~s}$, and FIB-4. ${ }^{41}$ In the combined model with a constant parameter, the AUROCs were sufficient: 0.87 for $\geq F 2$ with FIB-4, 0.89 for $\geq F 3$ with $T E$, and 0.99 for cirrhosis with TE. Therefore, the contrast parameter may be promising for grading hepatic fibrosis when combined with liver stiffness or FIB-4.

\section{Assessment of captured microbubbles}

Sonazoid is the only second-generation contrast agent available for the abdomen with the accumulating property in the reticuloendothelial system. The post-vascular phase signal intensity from the intrahepatic accumulated microbubbles showed AUROCs of 0.88 for $\geq F 2,0.95$ for $\geq F 3$, and 0.97 for cirrhosis in 203 subjects. ${ }^{42}$ The results were superior to those obtained with FIB-4 ( 0.85 for $\geq F 2, P=0.15 ; 0.89$ for $\geq F 3, P=0.057 ; 0.90$ for cirrhosis, $P=0.017)$. From a technical aspect, it appears easier to conduct scanning and to assess the findings with the captured microbubbles compared to the dynamic microbubbles, because of the stability of the target. Also, it should be recognized that there is a difference in the origin of signal source between SonoVue and Sonazoid when interpreting the data because the former does not accumulate in the liver.

\section{Differentiation between idiopathic portal hypertension (IPH) and cirrhosis}

Although it may not be a common disease, IPH causes several manifestations of portal hypertension, gastroesophageal varices, ascites, and a higher incidence of developing portal vein thrombosis. ${ }^{43} \mathrm{IPH}$ should be strictly distinguished from cirrhosis because of the differences in clinical management, the lower incidence of developing into HCC, and a better survival rate than cirrhosis. ${ }^{43}$ For this purpose, contrast-enhanced US with Sonazoid is effective to identify IPH by means of earlier-phase appearance of the liver showing delayed periportal enhancement, ${ }^{44}$ degree of post-vascular phase enhancement by accumulated microbubbles in the liv$\mathrm{er}^{45}$ and demonstration of unique structure of the intrahepatic portal vein by $3 \mathrm{D}$ imaging. ${ }^{46}$

\section{Severity of portal hypertension}

The transit time between vessels could be applicable to predict the severity of portal hypertension. A large study reported that the AUROC of HVAT (SonoVue) was 0.973 for clinically significant portal hypertension (HVPG $>10 \mathrm{mmHg}$ ) in compensated cirrhosis under the cut-off value of 14 seconds. ${ }^{47}$ The other study also reported that intrahepatic transit time under 6 seconds showed the diagnostic ability for SPH (HVPG $>12 \mathrm{mmHg}$ ) with AUROC of $0.94{ }^{48}$ A more recent study reported that splenic circulation presented by the transit time from the splenic artery to splenic vein using Sonazoid was an effective marker for the severity of portal hypertension. ${ }^{49}$

\section{SUMMARY AND PROSPECTIVE}

The pieces of evidence support the benefit of US in the diagnosis of chronic liver diseases, regarding the characterization of liver diseases and the assessment of the severity of fibrosis and portal hemodynamics. However, there are some limitations. Firstly, skill and knowledge of the US depend on the experience and may affect the operation and interpretation of the findings. Particularly, achievement of the valid data may be difficult in obese patients even by the trained person. Secondly, there are not enough studies to compare the diagnostic abilities of US and other imaging modalities, such as elastography and magnetic resonance imaging (MRI). Additional studies, including an international multicenter setting from different races and clinical backgrounds, may be nec- 
Hitoshi Maruyama, et al. Ultrasound in chronic liver diseases

essary to solve these problems.

There are two novel techniques in the abdominal US field. One is the fusion imaging which is the combined multiple imaging of US, CT, and MRI. Although it is generally used for the support of treating liver tumors, ${ }^{50}$ the role in the diagnosis of chronic liver disease is not determined. The second is the radiomics by means of the high-throughput extraction of large amounts of image features from radiographic images. This technology, based on automated and reproducible analysis, may suggest the beginning of the new era in the imaging field of chronic liver disease, though further validation study is needed. ${ }^{51-53}$

\section{CONCLUSION}

This article demonstrated the current application, substantial effects, and limitation of US in the diagnosis of chronic liver diseases. Further validation in a large-scale prospective study may enhance the establishment of US-based standard medical care of patients with chronic liver diseases.

\section{Authors' contributions}

Interpreting data: Naoya Kato.

Drafting the manuscript: Hitoshi Maruyama.

Final approval: All authors.

\section{Conflicts of Interest}

The authors have no conflicts to disclose.

\section{REFERENCES}

1. Williams R. Global challenges in liver disease. Hepatology 2006:44:521-526.

2. Cárdenas A, Ginès P. Management of patients with cirrhosis awaiting liver transplantation. Gut 2011;60:412-421.

3. Nissen NN, Martin P. Hepatocellular carcinoma: the high-risk patient. J Clin Gastroenterol 2002;35(5 Suppl 2):S79-S85.

4. Tandon P, Garcia-Tsao G. Portal hypertension and hepatocellular carcinoma: prognosis and beyond. Clin Gastroenterol Hepatol 2006:4:1318-1319.

5. Kim MY, Jeong WK, Baik SK. Invasive and non-invasive diagnosis of cirrhosis and portal hypertension. World J Gastroenterol 2014;20:4300-4315.

6. Baik SK. Haemodynamic evaluation by Doppler ultrasonography in patients with portal hypertension: a review. Liver Int 2010;30:1403-1413.

7. Maruyama H, Shiha G, Yokosuka O, Kumar A, Sharma BC, Ibrahim $A$, et al. Non-invasive assessment of portal hypertension and liver fibrosis using contrast-enhanced ultrasonography. Hepatol Int 2016;10:267-276.

8. Oberti F, Valsesia E, Pilette C, Rousselet MC, Bedossa P, Aubé C, et al. Noninvasive diagnosis of hepatic fibrosis or cirrhosis. Gastroenterology 1997;113:1609-1616.

9. Colli A, Fraquelli M, Andreoletti M, Marino B, Zuccoli E, Conte D. Severe liver fibrosis or cirrhosis: accuracy of US for detection--analysis of 300 cases. Radiology 2003;227:89-94.

10. Maruyama H, Yokosuka O. Ultrasonography for noninvasive assessment of portal hypertension. Gut Liver 2017;11:464-473.

11. Montano-Loza AJ, Meza-Junco J, Prado CM, Lieffers JR, Baracos VE, Bain VG, et al. Muscle wasting is associated with mortality in patients with cirrhosis. Clin Gastroenterol Hepatol 2012;10:166-173.

12. Periyalwar $P$, Dasarathy $S$. Malnutrition in cirrhosis: contribution and consequences of sarcopenia on metabolic and clinical responses. Clin Liver Dis 2012;16:95-131.

13. Baumgartner RN, Koehler KM, Gallagher D, Romero L, Heymsfield $\mathrm{SB}$, Ross RR, et al. Epidemiology of sarcopenia among the elderly in New Mexico. Am J Epidemiol 1998;147:755-763.

14. Tandon P, Ney M, Irwin I, Ma MM, Gramlich L, Bain VG, et al. Severe muscle depletion in patients on the liver transplant wait list: its prevalence and independent prognostic value. Liver Transpl 2012;18:1209-1216.

15. Englesbe MJ, Patel SP, He K, Lynch RJ, Schaubel DE, Harbaugh C, et al. Sarcopenia and mortality after liver transplantation. J Am Coll Surg 2010;211:271-278.

16. Meza-Junco J, Montano-Loza AJ, Baracos VE, Prado CM, Bain VG, Beaumont $C$, et al. Sarcopenia as a prognostic index of nutritional status in concurrent cirrhosis and hepatocellular carcinoma. J Clin Gastroenterol 2013;47:861-870.

17. Kaido T, Ogawa K, Fujimoto Y, Ogura Y, Hata K, Ito T, et al. Impact of sarcopenia on survival in patients undergoing living donor liver transplantation. Am J Transplant 2013;13:1549-1556.

18. Kobayashi K, Maruyama H, Kiyono S, Ogasawara S, Suzuki E, Ooka $Y$, et al. Application of transcutaneous ultrasonography for the diagnosis of muscle mass loss in patients with liver cirrhosis. J Gastroenterol 2018;53:652-659.

19. Sekimoto T, Maruyama H, Kondo T, Shimada T, Takahashi M, Yokosuka 0 , et al. Virtual laparoscopy: initial experience with three-dimensional ultrasonography to characterize hepatic surface features. Eur J Radiol 2013;82:929-934.

20. European Association for the Study of the Liver. EASL Clinical Practice Guidelines: vascular diseases of the liver. J Hepatol 2016;64:179-202.

21. Kim MY, Baik SK, Park DH, Lim DW, Kim JW, Kim HS, et al. Damping 
index of Doppler hepatic vein waveform to assess the severity of portal hypertension and response to propranolol in liver cirrhosis: a prospective nonrandomized study. Liver Int 2007;27:1103-1110.

22. Kondo T, Maruyama H, Sekimoto T, Shimada T, Takahashi M, Yokosuka O. Reversed portal flow: clinical influence on the long-term outcomes in cirrhosis. World J Gastroenterol 2015;21:8894-8902.

23. Kondo T, Maruyama H, Sekimoto T, Shimada T, Takahashi M, Okugawa $\mathrm{H}$, et al. Impact of portal hemodynamics on doppler ultrasonography for predicting decompensation and long-term outcomes in patients with cirrhosis. Scan J Gastroenterol 2016;51:236-244.

24. Maruyama H, Kobayashi K, Kiyono S, Ogasawara S, Ooka Y, Suzuki $E$, et al. Left gastric vein-based noninvasive test for esophageal varices: a same-day comparison of portal hemodynamic assessment with endoscopic appearance. Clin Transl Gastroenterol 2018;9:154.

25. Garcia-Pagán JC, Barrufet M, Cardenas A, Escorsell A. Management of gastric varices. Clin Gastroenterol Hepatol 2014;12:919-928.e1; quiz e51-e52.

26. Maruyama H, Kamezaki H, Kondo T, Sekimoto T, Shimada T, Takahashi $M$, et al. The application of transabdominal 3D ultrasound for the diagnosis of gastric varices: a preliminary study. Eur J Radiol 2013;82:e400-e404.

27. Maruyama H, Kamezaki H, Takahashi M, Okugawa H, Yokosuka 0. The potential of transabdominal 3D color doppler ultrasonography for diagnosis of gastric varices. J Clin Gastroenterol 2014;48:629-634.

28. Maruyama H, Ishihara T, Ishii H, Tsuyuguchi T, Yoshikawa M, Matsutani $S$, et al. Blood flow parameters in the short gastric vein and splenic vein on Doppler ultrasound reflect gastric variceal bleeding. Eur J Radiol 2010;75:e41-e45.

29. Maruyama H, Okugawa H, Yoshizumi H, Kobayashi S, Yokosuka O. Hemodynamic features of gastrorenal shunt: a Doppler study in cirrhotic patients with gastric fundal varices. Acad Radiol 2008;15:1148-1154.

30. Maruyama H, Okugawa H, Kobayashi S, Yoshizumi H, Yokosuka 0. Pre-treatment hemodynamic features involved with long-term survival of cirrhotic patients after embolization of gastric fundal varices. Eur J Radiol 2010;75:e32-e37.

31. Kamezaki H, Maruyama H, Shimada T, Takahashi M, Okugawa $H$, Yokosuka O. Short- and long-term clinical outcome after balloonoccluded retrograde transvenous obliteration: is pretreatment portal flow direction a predictive factor? Hepatol Int 2013;7:241-247.

32. Claudon $M$, Dietrich CF, Choi BI, Cosgrove DO, Kudo M, Nolsøe CP, et al. Guidelines and good clinical practice recommendations for Contrast Enhanced Ultrasound (CEUS) in the liver - update 2012: a WFUMB-EFSUMB initiative in cooperation with representatives of AFSUMB, AIUM, ASUM, FLAUS and ICUS. Ultrasound Med Biol 2013;39:187-210

33. Cobbold JF, Patel D, Fitzpatrick JA, Patel N, Crossey MM, Abdalla $M S$, et al. Accuracy and reliability of microbubble ultrasound mea- surements for the non-invasive assessment of hepatic fibrosis in chronic hepatitis C. Hepatol Res 2012;42:515-522.

34. Staub F, Tournoux-Facon C, Roumy J, Chaigneau C, MorichautBeauchant $\mathrm{M}$, Levillain $\mathrm{P}$, et al. Liver fibrosis staging with contrastenhanced ultrasonography: prospective multicenter study compared with METAVIR scoring. Eur Radiol 2009;19:1991-1997.

35. Li N, Ding H, Fan P, Lin X, Xu C, Wang W, et al. Intrahepatic transit time predicts liver fibrosis in patients with chronic hepatitis $B$ : quantitative assessment with contrast-enhanced ultrasonography. Ultrasoud Med Biol 2010;36:1066-1075.

36. Cobbold JF, Crossey MM, Colman P, Goldin RD, Murphy PS, Patel N, et al. Optimal combinations of ultrasound-based and serum markers of disease severity in patients with chronic hepatitis C. J Viral Hepat 2010;17:537-545.

37. Tang A, Kim TK, Heathcote J, Guindi M, Jang HJ, Karshafian R, et al. Does hepatic vein transit time performed with contrast-enhanced ultrasound predict the severity of hepatic fibrosis? Ultrasound Med Biol 2011;37:1963-1969.

38. Ridolfi F, Abbattista T, Marini F, Vedovelli A, Quagliarini P, Busilacchi $P$, et al. Contrast-enhanced ultrasound to evaluate the severity of chronic hepatitis C. Dig Liver Dis 2007;39:929-935.

39. Orlacchio A, Bolacchi F, Petrella MC, Pastorelli D, Bazzocchi G, Angelico $M$, et al. Liver contrast enhanced ultrasound perfusion imaging in the evaluation of chronic hepatitis $C$ fibrosis: preliminary results. Ultrasound Med Biol 2011;37:1-6.

40. Ishibashi H, Maruyama H, Takahashi M, Fujiwara K, Imazeki F, Yokosuka 0 . Assessment of hepatic fibrosis by analysis of the dynamic behavior of microbubbles during contrast ultrasonography. Liver Int 2010;30:1355-1363.

41. Tawada A, Maruyama H, Kamezaki H, Shimada T, Ishibashi H, Takahashi $M$, et al. Magnitude of contrast-enhanced ultrasonography as a noninvasive predictor for hepatic fibrosis: comparison with liver stiffness measurement and serum-based models. Hepatol Int 2013;7:749-757.

42. Ishibashi H, Maruyama H, Takahashi M, Shimada T, Kamesaki H, Fujiwara K, et al. Demonstration of intrahepatic accumulated microbubble on ultrasound represents the grade of hepatic fibrosis. Eur Radiol 2012;22:1083-1090.

43. Maruyama H, Kondo T, Sekimoto T, Yokosuka O. Differential clinical impact of ascites in cirrhosis and idiopathic portal hypertension. Medicine (Baltimore) 2015;94:e1056.

44. Maruyama H, Shimada T, Ishibashi H, Takahashi M, Kamesaki H, Yokosuka O. Delayed periportal enhancement: a characteristic finding on contrast ultrasound in idiopathic portal hypertension. Hepatol Int 2012;6:511-519.

45. Maruyama H, Ishibashi H, Takahashi M, Imazeki F, Yokosuka O. Effect of signal intensity from the accumulated microbubbles in the liver for differentiation of idiopathic portal hypertension from liver 
Hitoshi Maruyama, et al.

Ultrasound in chronic liver diseases

cirrhosis. Radiology 2009;252:587-594.

46. Maruyama H, Okugawa H, Kobayashi S, Yoshizumi H, Takahashi M,

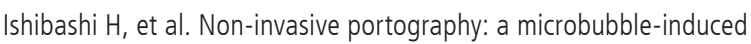
three-dimensional sonogram for discriminating idiopathic portal hypertension from cirrhosis. Br J Radiol 2012;85:587-595.

47. Kim MY, Suk KT, Baik SK, Kim HA, Kim YJ, Cha SH, et al. Hepatic vein arrival time as assessed by contrast-enhanced ultrasonography is useful for the assessment of portal hypertension in compensated cirrhosis. Hepatology 2012;56:1053-1062.

48. Jeong WK, Kim TY, Sohn JH, Kim Y, Kim J. Severe portal hypertension in cirrhosis: evaluation of perfusion parameters with contrastenhanced ultrasonography. PLoS One 2015;10:e0121601.

49. Shimada T, Maruyama H, Kondo T, Sekimoto T, Takahashi M, Yokosuka O. Impact of splenic circulation: non-invasive microbubble- based assessment of portal hemodynamics. Eur Radiol 2015;25:812 820.

50. Maruyama H, Sekimoto T, Yokosuka O. Role of contrast-enhanced ultrasonography with sonazoid for hepatocellular carcinoma: evidence from a 10-year experience. J Gastroenterol 2016;51:421-433.

51. Lambin P, Leijenaar RTH, Deist TM, Peerlings J, de Jong EEC, van Timmeren J, et al. Radiomics: the bridge between medical imaging and personalized medicine. Nat Rev Clin Oncol 2017;14:749-762.

52. Gillies RJ, Kinahan PE, Hricak H. Radiomics: images are more than pictures, they are data. Radiology 2016;278:563-577.

53. Guo Y, Hu Y, Qiao M, Wang Y, Yu J, Li J, et al. Radiomics analysis on ultrasound for prediction of biologic behavior in breast invasive ductal carcinoma. Clin Breast Cancer 2018;18:e335-e344. 